\title{
EN LA UNIVERSIDAD, ¿CÓMO MEJORAR LA EVALUACIÓN FORMATIVA MEDIANTE EL USO DE LAS TECNOLOGÍAS DE LA INFORMACIÓN Y LA COMUNICACIÓN?
}

\author{
How to improve formative evaluation with Information and Communication \\ Technology at university?
}

Como melhorar a avaliação formativa, através das Tecnologias da Informação e

Comunicação na universidade?

\begin{abstract}
Marta Fuentes Agustí (1)
Aportación derivada del proyecto: “¿Nos pueden ayudar las tecnologías a una evaluación formativa sostenible?". Oficina de Calidad Docente de la Universidad Autónoma de Barcelona (OQD-UAB): Proyectos de soporte a la innovación docente convocatoria 2017.

(1) Universidad Autónoma de Barcelona, España. Teléfono: +34 935812627. marta.fuentes@uab.cat
\end{abstract}

\section{Resumen}

La intención de este texto es promover el conocimiento y difusión de experiencias y estudios relacionados con el uso de las Tecnologías de la Información y la Comunicación en la Evaluación Formativa universitaria. El método seguido consiste en compartir experiencias, repensar la propia docencia, formarse conjuntamente, diseñar, implementar, co-evaluar y transformar. Los resultados muestran diversidad de recursos y herramientas tecnológicas que ayudan a desarrollar una evaluación formativa de calidad y sostenible. Se ha alcanzado un buen rendimiento académico de los estudiantes basado en la mejora de la comunicación y acompañamiento, se ha reducido el tiempo destinado a la gestión docente, motivado para el cambio sin temores, transferido experiencias a otras prácticas, impulsado la reflexión comparativa y la autorregulación. Se concluye resaltando la necesidad de compartir al resto de la comunidad estrategias e instrumentos de buenas prácticas de evaluación-aprendizaje con TIC junto con indicaciones sobre su elección y uso.

Palabras clave: Evaluación formativa; tecnologías; estudiante; práctica docente; Universidad

Abstract
The intention of this paper is to promote knowledge and dissemination of experiences and
studies related to the use of Information and Communication Technologies in the
En la universidad, ¿cómo mejorar la evaluación formativa mediante el uso de las tecnologías de la
información y la comunicación? 
University Formative Assessment. Our methodology consisted in sharing experiences, rethinking teaching, training together, and designing, implementing, peer evaluating and transforming our teaching. The results show a diversity of resources and technological tools that help to develop a sustainable and quality formative assessment. A good academic performance of the students has been achieved based on the improvement of the communication and tutoring, a reduction of the time allocated to teaching management, the motivation for no fear of change, the transfer of experiences to other practices, the promotion of comparative reflection and the self-regulation. Findings conclude that there is need to share with the rest of the community strategies and instruments of good practices of evaluation-learning with ICT, and create a series of indications about their choice and their use.

Keywords: Formative evaluation; technologies; student; teaching practice; University

\section{Resumo}

Este texto pretende promover o conhecimento e a divulgação de experiências e estudos relacionados com o uso das Tecnologias de Informação e Comunicação na Avaliação Formativa Universitária. O método seguido consiste na partilha de experiências, repensar a própria docência, formação em conjunto, desenhar, implementar, coavaliar e transformar. Os resultados mostram uma diversidade de recursos e ferramentas tecnológicas que ajudam a desenvolver uma avaliação formativa sustentável e de qualidade. Alcançou-se um bom desempenho académico dos alunos, face a uma melhoria na comunicação e acompanhamento, redução do tempo destinado à gestão docente, motivação para a mudança, sem medos, transferindo experiências para outras práticas, promovendo a reflexão comparativa e a autorregulação. Conclui-se, salientado a necessidade de partilhar com o resto da comunidade, estratégias e instrumentos de boas práticas de avaliação-aprendizagem com as TIC, bem como indicações sobre como escolher e usar.

Palavras-chave: Avaliação formativa; tecnologias; estudante; prática docente; Universidade

\section{Introducción}

La intención de este texto es promover el conocimiento y difusión de experiencias y estudios realizados por los componentes del Grupo de Innovación sobre el Portafolio 
Docente (Gi-PD) de la Universidad Autónoma de Barcelona (UAB). Dicho grupo en los dos últimos cursos académicos ha analizado el uso que hacen y pueden hacer de las Tecnologías de la Información y la Comunicación (TIC) en la Evaluación Formativa y Compartida (EFC) impulsada desde esta institución. El grupo consta de docentes noveles y seniors de diferentes áreas de conocimientos motivados por la innovación y la calidad docente.

La metodología habitual seguida por el grupo en sus encuentros se basa en la socialización y el debate con componentes propios de la práctica reflexiva con transformaciones agentivas. Por un lado, se adopta una actitud de aprendices a lo largo de toda la trayectoria profesional (Ibáñez, 1990; Esteve, Melief \& Alsina, 2010) estando siempre latente la reflexión (Perrenoud, 2004), la autoevaluación (Ramos \& Rodríguez, 2000; Rebolloso \& Pozo, 2000), la asistencia a congresos y seminarios de intercambios de experiencias (Mayor, 1997) y la formación permanente (Imbernón, 2007; Pozo, 2008). Y por otro, se parte de la premisa que la actuación docente se produce en un "marco de condiciones" (Zabalza, 2003) y retos (Quintanal, 2003).

Los tres últimos años se ha optado por trabajar en profundidad el binomio Evaluación y TIC, por su incidencia en la formación y profesionalización de los estudiantes universitarios sea cuál sea su campo de estudio. Las formaciones y filosofías docentes de partida de los componentes del grupo eran muy diversas así como las prácticas desarrolladas hasta el momento. Por lo que, en primera instancia, quisimos distinguir entre las dos principales funciones de la evaluación académica de los estudiantes: la formativa-formadora y la acreditativa (Figura 1).

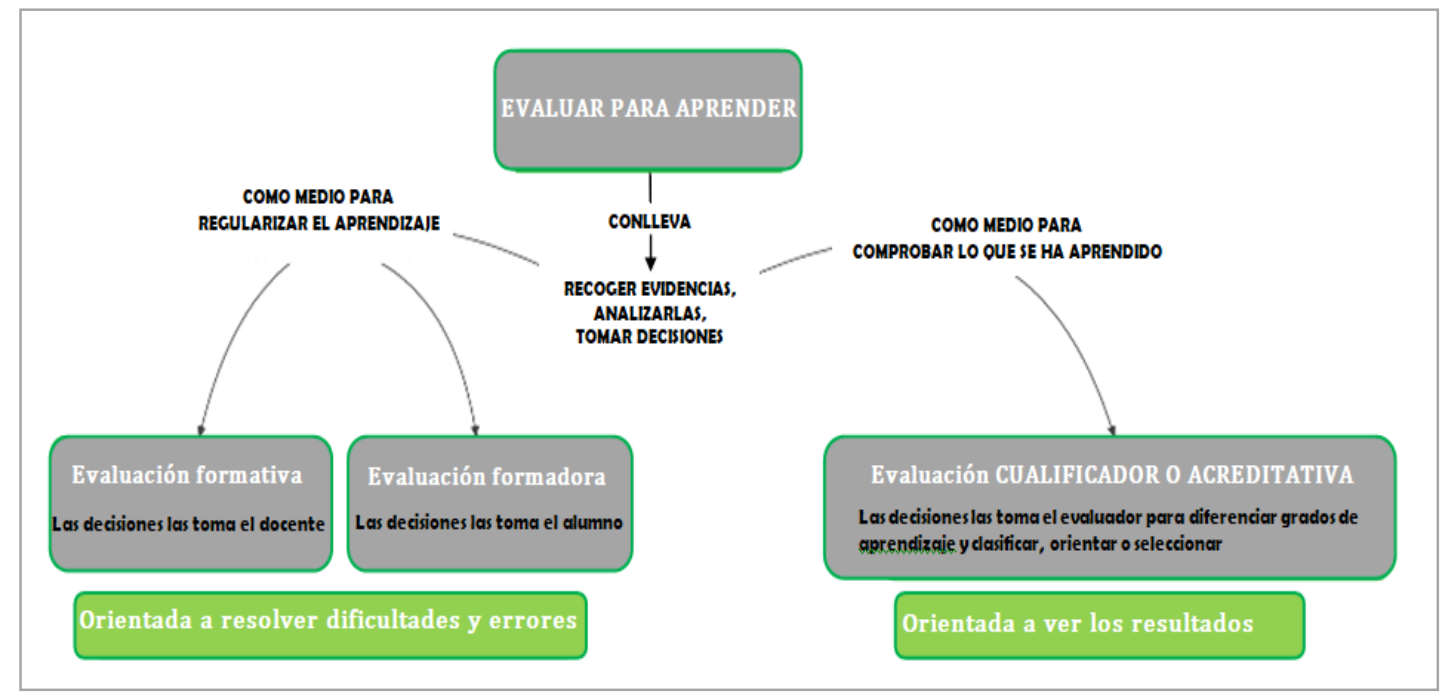

Figura 1. Funciones de la evaluación académica.

En la universidad, ¿cómo mejorar la evaluación formativa mediante el uso de las tecnologías de la información y la comunicación? 
Entendemos que la aplicación de sistemas de evaluación formativa a los procesos educativos que se dan en las aulas (tradicionales y extendidas) es una pieza clave para el logro de un mayor aprendizaje, especialmente en los modelos fomentados por el Espacio Europeo de Educación Superior (EEES) centrados en el estudiante y el desarrollo de competencias (Andrade \& Cizek, 2010; Fuentes, 2017; Lopez-Pastor \& Pérez-Pueyo, 2017).

\section{Método y resultados}

Fase I: A lo largo del curso 2017-18 se desarrolló un proyecto denominado “¿Nos pueden ayudar las tecnologías a una evaluación formativa sostenible?" que partió de una encuesta donde se recogían datos sobre la situación inicial de cada uno de los docentes participantes (Figura 2).

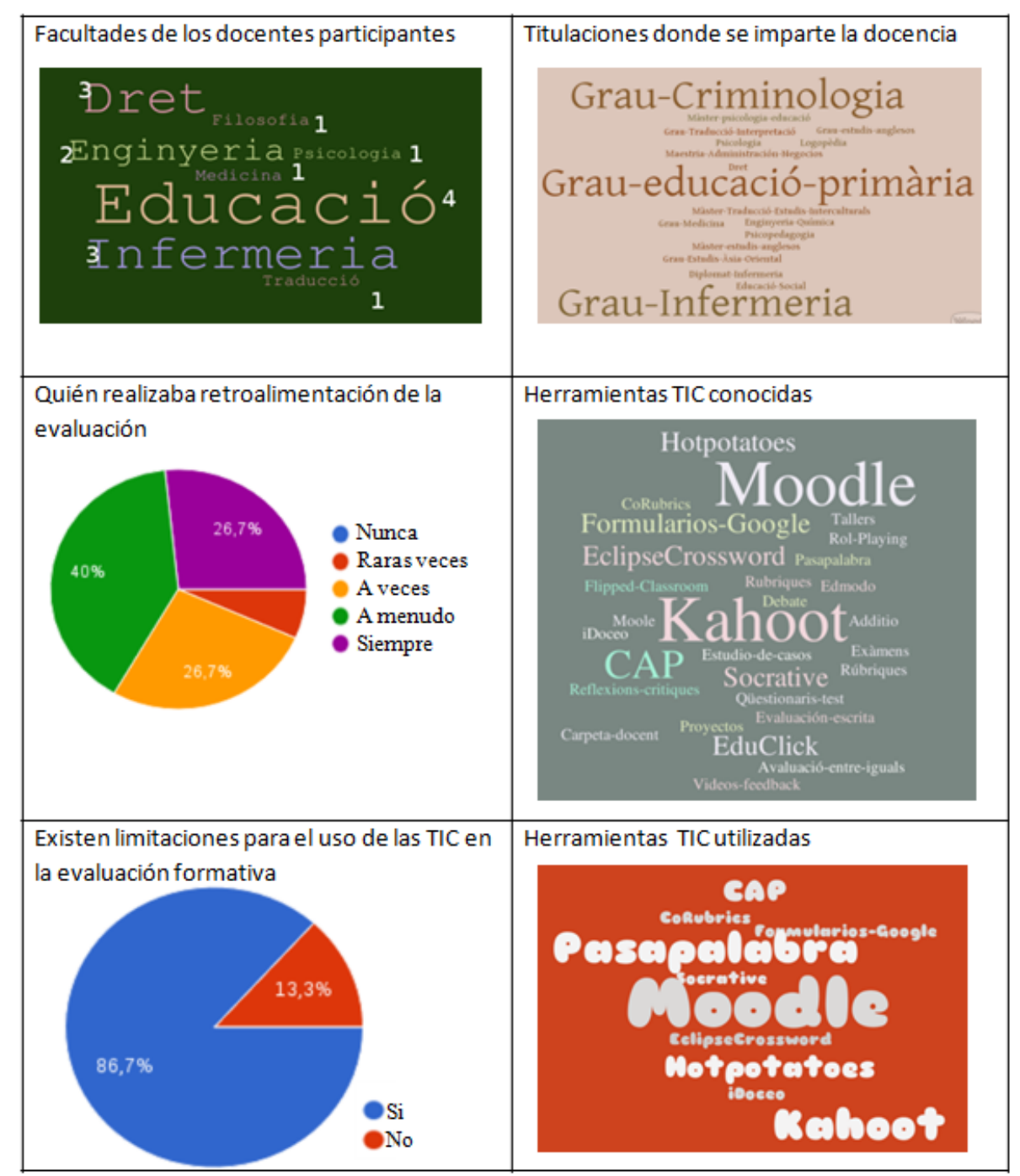

Figura 2. Perfil inicial del grupo de docentes participantes en la experiencia. Datos recogidos en octubre de 2017.

En la universidad, ¿cómo mejorar la evaluación formativa mediante el uso de las tecnologías de la información y la comunicación? 
Las limitaciones citadas por los docentes hacían referencia a: falta de tiempo (1), de formación (2) o de presupuesto (3), desacuerdo entre compañeros (4), poca autonomía del profesor que imparte la asignatura -guías docentes no elaboradas por ellos- (5), normativa astringente (6) y otras particularidades (7) tales como la inestabilidad de las conexiones en las aulas y la incompatibilidad de los programas instalados en los dispositivos de los despachos y aulas (Figura 3).

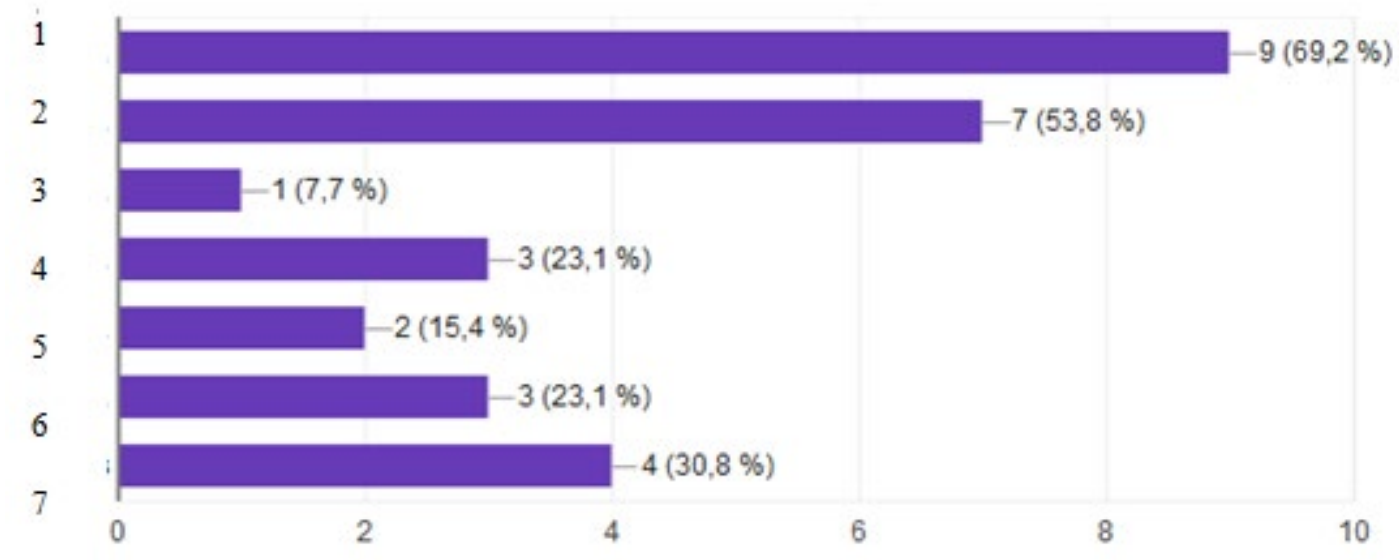

Figura 3. Distribución de las limitaciones citadas por los docentes. Datos recogidos en octubre de 2017.

Este perfil es muy diferente al actual. Se ha observado un impacto positivo de la segunda fase del proyecto.

Fase II: A continuación se compartieron y analizaron prácticas docentes, recursos e instrumentos digitales concretos de evaluación de los estudiantes (y por ende, de aprendizaje) con la finalidad de crecer juntos (formarse y tomar apuntes de mejora) para conseguir desarrollar a corto-medio plazo una evaluación formativa-formadora de calidad y al mismo tiempo sostenible. Las prácticas docentes compartidas fueron analizadas mediante actas de encuentros y relatos elaborados por los docentes los cuales se han contrastado con las aportaciones de los estudiantes (cuestionarios de satisfacción, gruposfoco y entrevistas) y, en algunos casos, los registros de los entornos y/o las notas de campos de observaciones entre colegas.

La autoevaluación, reflexión individual-grupal y repensar la propia práctica docente han sido acciones constantes desarrolladas por los docentes implicados. Estas acciones se complementaron con formaciones entre compañeros y formaciones externas autogestionadas por el grupo. El análisis de las necesidades formativas y de las posibilidadeslimitaciones de incluir las TIC en las propias evaluaciones formativas, así como la 
necesidad inmediata de elegir-descartar recursos y herramientas en el diseño docente de las materias a impartir, ha propiciado la elaboración de orientaciones y pautas de uso (Figura 4).

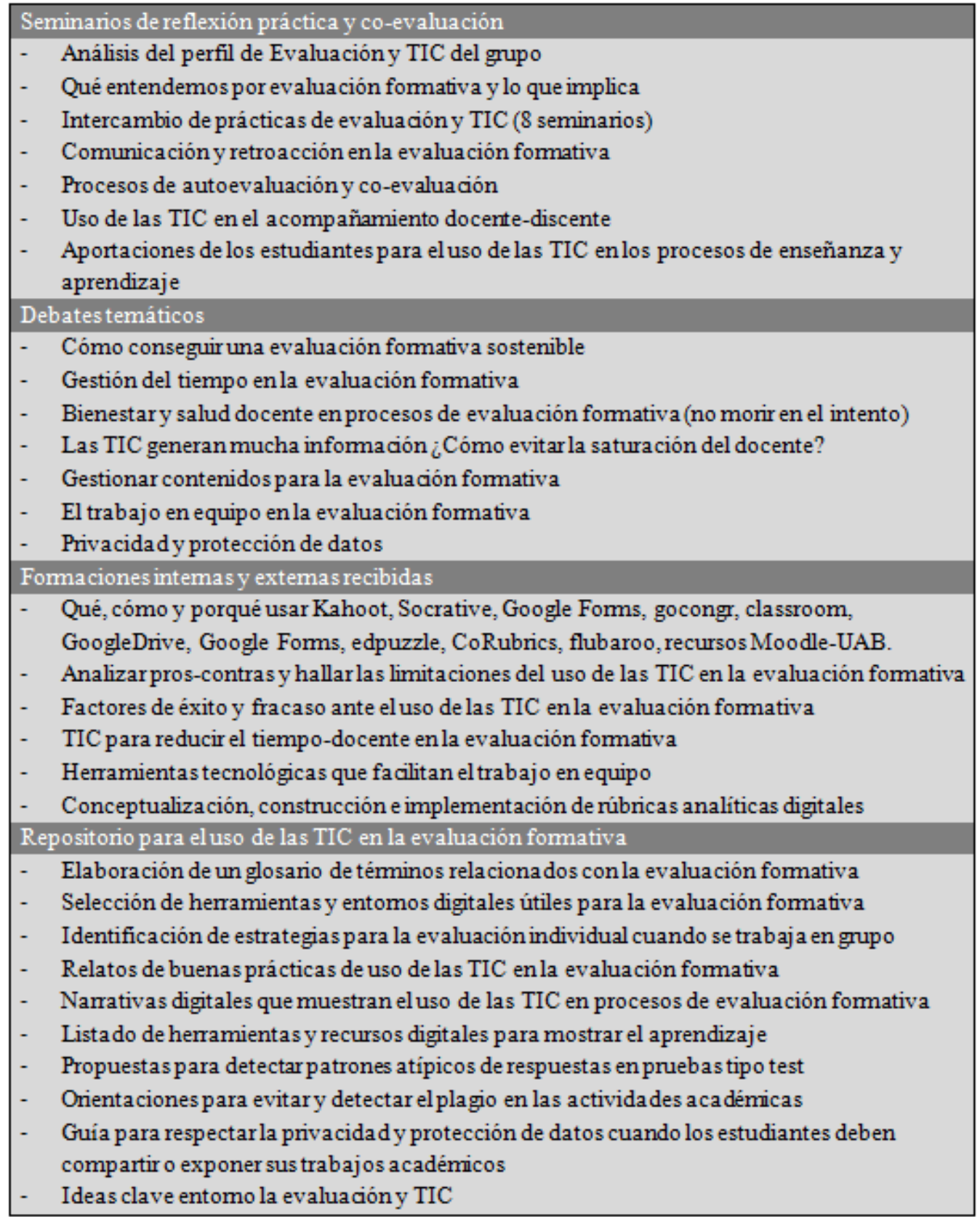

Figura 4. Resumen de las principales actividades con impacto positivo realizadas entre octubre de $2017 \mathrm{y}$ diciembre 2018.

Los resultados alcanzados indican que los docentes universitarios:

a) Debemos formarnos antes de implementar una evaluación formativa y compartida.

Tenemos carencias en el momento de definir este sistema de evaluación y, sobretodo, en cómo podemos implementarla.

En la universidad, ¿cómo mejorar la evaluación formativa mediante el uso de las tecnologías de la información y la comunicación? 
b) Tenemos problemas de sostenibilidad en relación con las tareas a realizar y el tiempo necesario vs disponible. Las TIC nos pueden ayudar pero para ello hay que conocerlas y seleccionarlas.

c) Desconocemos gran parte del amplio abanico existente de tecnologías que se pueden usar para el aprendizaje fomentando la evaluación formativa. Incluso, en algunos casos, este conocimiento es prácticamente nulo.

d) Disponemos de pocos criterios objetivos para la elección de las herramientas y entornos digitales más adecuados para la práctica docente que queremos llevar a cabo.

e) Consideramos las tecnologías un medio (no una finalidad) imprescindible a pesar que sus conocimientos teórico-práctico para implementar a una evaluación de calidad con TIC son insuficientes.

f) Pueden usar las TIC para la gestión, la planificación, el diseño, el desempeño, la evaluación, la recogida de evidencias, la comunicación de resultados de aprendizaje, la introducción de retroacción, etc. (Fuentes, 2018; Fuentes \& Valdivia, 2018).

\section{Conclusiones}

Los resultados indican que la manera idónea de hacer frente al reto de una evaluación formativa-formadora sostenible para el docente y los estudiantes es mediante la inclusión de una evaluación que fomente la autoevaluación y la evaluación compartida. Para ello se precisa de pautas socio-psicopedagógicas y organizativo-didácticas diversas que ayuden a la selección e implementación de las TIC en acorde con las distintas disciplinas que conforman una titulación.

Los docentes precisan conocer experiencias diversas por lo que se sugiere fomentar prácticas de intercambio, formaciones entre compañeros, formaciones externas a medida para el grupo de asistentes, participar en congresos de innovación educativa, leer artículos científicos y relatos publicados en las redes sociales. Todo ello acompañado de procesos de investigación, auto y co-evaluación, reflexión y transformación.

\section{Referencias}

Andrade, H. L. \& Cizek, G. J. (Eds.) (2010). Handbook of formative assessment. New York: Routledge.

Esteve, O., Melief, K. \& Alsina, A. (2010). Creando mi profesión. Una propuesta para el desarrollo profesional del profesorado. Barcelona: Octaedro.

En la universidad, ¿cómo mejorar la evaluación formativa mediante el uso de las tecnologías de la 
Fuentes, M. (2017). Evaluación y TIC. Material didáctico de La Universidad Internacional de Valencia: Máster en educación, tecnologías e innovación (TIC).

Fuentes, M. (2018). Herramientas y recursos digitales para el docente, el estudiante o el empresario basados en las tecnologías de la información y la comunicación. Publicado en https://ddd.uab.cat/pub/recdoc/2018/189284/DDD_HerramientasTIC_mfuentes2 018.pdf

Fuentes, M., \& Valdivia, P. (2018). Aportación del Moodle-UAB en la evaluación del estudiante universitario: reflexiones desde la praxis docente. En López-Meneses, E., Cobos-Sanchiz, D., Martín-Padilla, A. H., Molina-García, L. \& Jaén-Martínez, A. Experiencias pedagógicas e innovación educativa. Aportaciones desde la praxis docente e investigadora (pp. 2556-2575). Madrid: Octaedro.

Ibáñez, J. A. (1990). Dimensiones de la competencia profesional del profesor de universidad. Revista Española de Pedagogía, 186, 239-255.

Imbernón, F. (2007). 10 Ideas clave. La formación permanente del profesorado. Nuevas ideas para formar en la innovación y el cambio. Barcelona: Graó.

López-Pastor V. M. \& Pérez-Pueyo, A. (coords.) (2017). Evaluación formativa y compartida en Educación: experiencias de éxito en todas las etapas educativas. León: Universidad de León.

Mayor, C. (1997). El perfeccionamiento de los profesores universitarios principiantes a juicio de sus alumnos. Revista interuniversitaria de Formación del Profesorado, 30,127-149.

Perrenoud, P. (2004). Diez nuevas competencias para enseñar. Barcelona: Graó.

Pozo, I. (2008). Aprendices y maestros: la psicología cognitiva del aprendizaje. Madrid: Alianza Editorial.

Quintanal, J. (coord.). (2003). Los retos de la educación en el siglo XXI. Madrid: CES Don Bosco - Edebé.

Ramos, J. L. \& Rodríguez García, L. M. (2000). Autoevaluación del profesorado: un reto para su desarrollo profesional y para la mejora de la calidad educativa en el siglo XXI. Actas del X Congreso INFAD, (pp. 856-860).

En la universidad, ¿cómo mejorar la evaluación formativa mediante el uso de las tecnologías de la información y la comunicación? 
Rebolloso, E. \& Pozo, C. (2000). Las actitudes de los estudiantes universitarios hacia sus profesores: implicaciones para la mejora de la calidad docente. Revista Psicología Educativa, 6 (1), 27-50.

Zabalza, M. A. (2003). Competencias docentes del profesorado universitario. Calidady desarrollo profesional. Madrid: Narcea.

En la universidad, ¿cómo mejorar la evaluación formativa mediante el uso de las tecnologías de la información y la comunicación? 